\title{
Effect of Mechanical Strain on the Optical Properties of Quantum Dots: Controlling Exciton Shape, Orientation, and Phase with a Mechanical Strain
}

\author{
Garnett W. Bryant, ${ }^{1, *}$ M. Zieliński, ${ }^{2}$ Natalia Malkova, ${ }^{1}$ James Sims,${ }^{3}$ W. Jaskólski, ${ }^{2}$ and Javier Aizpurua ${ }^{4}$ \\ ${ }^{1}$ Atomic Physics Division and Joint Quantum Institute, National Institute of Standards and Technology, \\ 100 Bureau Drive, Gaithersburg, Maryland 20899-8423, USA \\ ${ }^{2}$ Instytut Fizyki UMK, Grudziadzka 5, 87-100 Toruń, Poland \\ ${ }^{3}$ Information Technology Laboratory, National Institute of Standards and Technology, \\ 100 Bureau Drive, Gaithersburg, Maryland 20899-8911, USA \\ ${ }^{4}$ Centro Mixto de Física de Materiales CSIC-UPV/EHU and Donostia International Physics Center, \\ Paseo Manuel Lardizabal 4, Donostia-San Sebastián 20018, Spain
}

(Received 22 March 2010; revised manuscript received 21 May 2010; published 5 August 2010)

\begin{abstract}
We show how a nanomechanical strain can be used to dynamically reengineer the optics of quantum dots, giving a tool to manipulate mechanoexciton shape, orientation, fine structure splitting, and optical transitions, transfer carriers between dots, and interact qubits for quantum processing. Most importantly, a nanomechanical strain reengineers both the magnitude and phase of the exciton exchange coupling to tune exchange splittings, change the phase of spin mixing, and rotate the polarization of mechanoexcitons, providing phase and energy control of excitons.
\end{abstract}

DOI: 10.1103/PhysRevLett.105.067404

PACS numbers: 78.67.Hc, 73.21.La, 85.35.Be

Quantum dots (QD) have attracted great interest for applications in photonics, sensors, quantum information, and precision measurement. Passive control of QDs is obtained by tailoring dot size, shape, and composition via growth. Dynamical control of exciton energies, polarization, and phase is highly desirable for QD nanophotonics. For example, a two photon cascade from the QD biexciton state can generate entangled photons in scalable devices [1]. However, anisotropic exchange splitting (AES) of QD excitons, induced by the asymmetry of typical as-grown QDs, inhibits entanglement. Annealing [2], magnetic, and electric fields [3-6], and dressing excitons with optical fields [7] are being used to modify AES. An imposed nanomechanical strain [8-11] provides a route to dynamically reengineer QD structural symmetry to control excitations, polarize transitions, tune exchange splitting, induce entanglement, or modify coupling between QDs. These are capabilities needed to use QDs in nanophotonics, quantum information processing, and in optically active devices, such as optomechanical cavities $[12,13]$ and semiconductor nanotubes [14-17].

Nanomechanics is being studied for mass sensing $[18,19]$, mechanical computing [20,21], and energy harvesting [22]. Structures are being cooled to approach the quantum limit for metrology and to provide coherent transducers that couple classical machines to quantum devices [23-26]. Surface acoustic waves (SAW) are being used to manipulate carriers in dynamically created QDs [27-32]. Local probes and control of nanomechanics and SAWs are needed. Sideband cooling via optical absorption by QDs in nanomechanical structures could drive structures to the quantum limit [25]. An experiment shows that QD levels are sensitive to a local strain produced by SAWs and mechanical deformations [8-11]. QD response could be a local strain gauge for nanomechanics.

To exploit hybrid nanomechanical-QD devices, a fundamental understanding is needed. This entails understanding connections between the strain from lattice mismatch, imposed nanomechanical strain, electron and hole states of QDs in the nanomechanical device, and strained excitons (mechanoexcitons) in excited QDs. We study pyramidal InAs QDs in a GaAs nanomechanical bridge using atomistic tight-binding theory. The bridge is bent to simulate an external strain applied to mechanoengineer QDs. A bend in a nanomechanical structure is analogous to an electric field, inducing Stark-like energy shifts. Electrons and holes redistribute vertically along the QD growth axis, or horizontally in the plane of the QD, depending on how strain is applied. This behavior correlates with bendinduced changes in the local band profile. Strain-induced charge redistribution in closely spaced QDs can induce tunneling between dots. Most importantly, nanomechanical strain reengineers the magnitude and the phase of the exciton exchange coupling via, primarily, strain-induced hole redistribution. This leads to large changes in exciton exchange splitting and polarization of bright excitons. Exciton phase control is achieved because the exchange reengineering changes the phase of spin mixing in the mechanoexcitons.

Using an atomistic model is critical for an accurate description of nanomechanical-QD hybrids with atomic scale variations in composition and shape, and significant local and imposed strain [33]. We use tight-binding theory [33-35] for electron and hole states with an $s p^{3} s^{*}$ orbital model, nearest-neighbor coupling, spin-orbit effects, strain from lattice mismatch, and imposed mechanical strain. 
Relaxation of local and imposed strain is included via atomistic valence force field theory. Exciton states are determined with a configuration-interaction treatment [36-38]. An atomistic model is essential for describing AES [39-41]. While AES arises if the QD has geometrical asymmetry [42], AES can arise even if the QD has in-plane geometrical symmetry, because the atomic lattice can break symmetry [39,40].

We consider a QD in a nanomechanical bridge, as shown in Fig. 1. The bridge is clamped at each end and bent along [100] by shifting the bridge vertically along [001]. After the structure is bent, surface atoms are held fixed while atoms inside the bridge relax to minimize imposed strain and lattice mismatch. The maximum bend amplitude $S$ we discuss produces a beam elongation of $0.25 \%$ and lattice distortions a tenth of the shifts due to lattice mismatch. For the simulations, the bridge is $80 \mathrm{~nm}$ wide in the lateral directions and $25 \mathrm{~nm}$ thick, with 10 million atoms. A small, square pyramidal QD (height $3 \mathrm{~nm}$, base $7 \mathrm{~nm}$ ) and the wetting layer are located near the middle of the bridge. The QD is symmetric, so AES is induced by atomic symmetry breaking. We consider two bends: a bend symmetric about the QD that distorts biaxial deformation from lattice mismatch, and a bend antisymmetric about the QD that gives vertical shear, as shown in Fig. 1. Our results are sensitive to bend geometry and dot location. Calculations for different bends, boundary conditions, and dot positions must be done to build a complete picture of nanomechanical strain effects $[8,11]$. We chose the bend along [100] to focus on effects of mechanical deformation rather than piezoelectric effects that can arise for other bend directions. For a small QD, piezoelectric effects are small [34]. We ignore these piezoelectric effects here.

QD electron energy shifts in a bent bridge are shown in Fig. 2. A symmetric bend raises (lowers) the electron energy when the bridge is bent up (down). Few meV shifts are possible for the $S$ shown. The $1 S-1 P$ splitting is nearly constant, suggesting that the main effect of the bend is a conduction-band profile shift. This bend has similar effects on hole levels. For a shearing bend, the lowest confined electron energies increase until they cross wetting layer states for $S \approx 2 a$ ( $a$ is the GaAs lattice constant), indicating that confined levels are shifted laterally out of the QD by shear. In contrast, a shearing bend reduces hole energies. As illustrated in Fig. 2, the band gap $E_{g}$ can increase or decrease substantially for symmetric bends, but $E_{g}$ decreases only slightly for shearing bends (because hole

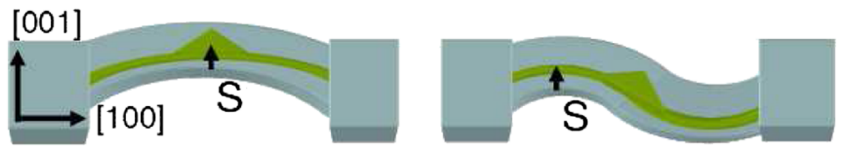

FIG. 1 (color online). Schematic of a bend applied to a QD in a nanobridge: (left) symmetric (biaxial deformation) bend with a QD at an antinode, and (right) vertical shearing bend with a QD at a node. $S$ is the bend amplitude for clamped ends. energies change more than electron energies). Figure 2 also shows how states redistribute $\left(\Delta \rho_{e, v}\right)$ inside the dot when the bridge is bent. A symmetric bend shifts both electrons and holes vertically. A shearing bend pushes electrons and holes horizontally in opposite directions. This spatial control is important for coupled dots. Calculations for a pair of QDs in a bridge show that a symmetric bend can transfer charge between vertically stacked QDs, while a shear bend can transfer charge between laterally coupled QDs.

Effects of mechanical strain can be explained by straininduced changes to band profiles [34,43]. For electrons, the change is primarily via the hydrostatic deformation potential. For holes, there is also a shear contribution. Here we discuss the simpler case for electrons. Figure 3 sketches the biaxial deformation of the QD due to lattice mismatch. When the bridge is bent symmetrically, internal reaction tries to undo the bend, providing vertical expansion (compression) near the wetting layer and compression (expansion) near the QD apex for $S<0(S>0)$. As shown in Fig. 3(d), this relaxation lowers (raises) the conduction band near the wetting layer and raises (lowers) the band near the QD apex for $S<0(S>0)$. The response is not a uniform band shift. Local variation of the QD band profile shifts the energies and redistributes electron density. The effect of a shearing bend on electrons arises from internal relaxation which pushes up one side of the dot and pushes down the other side in reaction to the bend, providing a local profile which is lower on one side of the dot and higher on the other.

Models for QDs coupled to mechanical strain which mechanically deform the QD without the internal reaction will be suspect. Calculations done by taking a flat beam with relaxation due to lattice mismatch, bending it, but not allowing additional reaction, predict energy shifts opposite to those in Fig. 2. The local, nonlinear lattice reaction must be included. In contrast, SAW structures with weaker strain

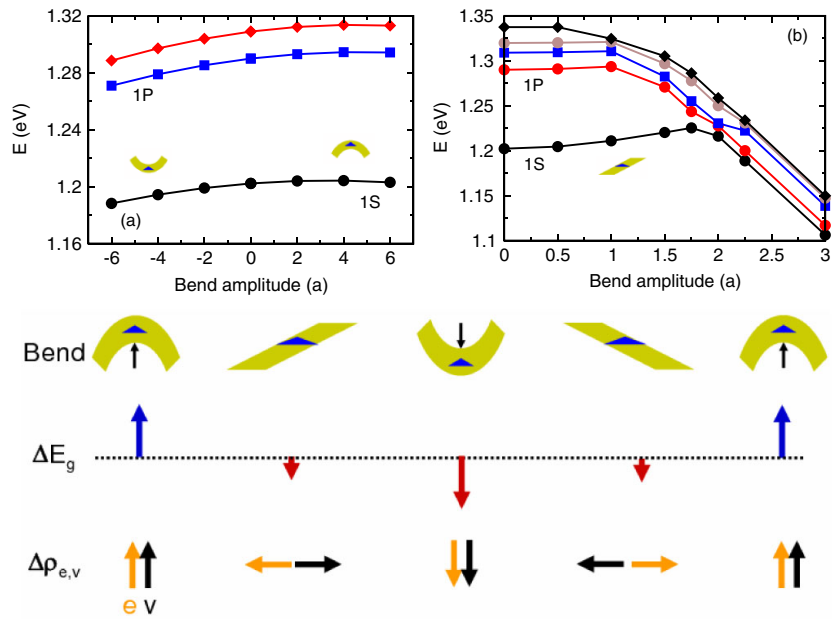

FIG. 2 (color online). Electron $1 S$ and $1 P$ energies for (a) a biaxial deformation and (b) a shearing bend. Blue or red band gap shift $\Delta E_{g}$ and redistribution $\Delta \rho_{e, v}$ of the lowest conduction $(e)$ and highest valence state $(v)$ for different bends. 
(a) Relaxation in a flat bridge

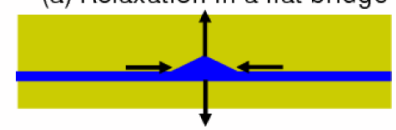

(b) Relaxation in a bent bridge

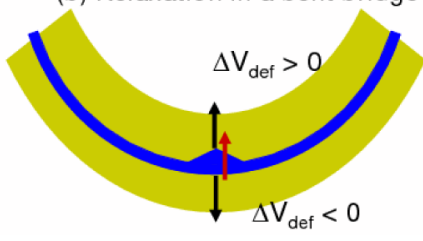

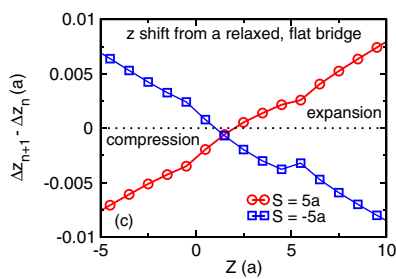

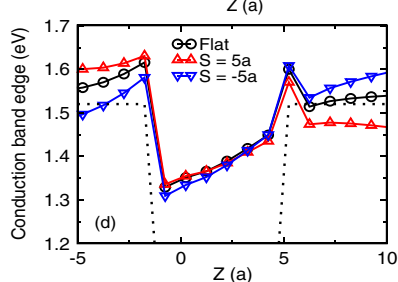

FIG. 3 (color online). Schematics for (a) relaxation due to lattice mismatch in a flat bridge and (b) additional relaxation [grey (red) arrow] in a bent bridge. (c) Lattice shift along the vertical axis through the QD center for biaxial deformation, $\Delta z_{n}$ is the extra relaxation of atom $n$ from its position in a flat bridge for atoms ordered along the axis, and (d) conduction band profile along the vertical axis for a flat bridge and biaxially deformed bridges. The dotted curve is the unrelaxed band profile.

do couple linearly to QDs and can be accounted for via band shifts (results not shown).

For the QDs considered, the lowest excitons are made from the lowest-energy electron-hole pair states with little mixing of higher pair states. The lowest electron and hole states are spin-degenerate, so the lowest pair state is fourfold degenerate. Coulomb and exchange interaction split the pair ground state into two lower-energy dark excitons (DE) and two bright excitons (BE). Figure 4 shows the energy splitting of these four mechanoexcitons from the lowest pair state. The two DEs are nearly degenerate and split from the pair ground state by the binding energy. The
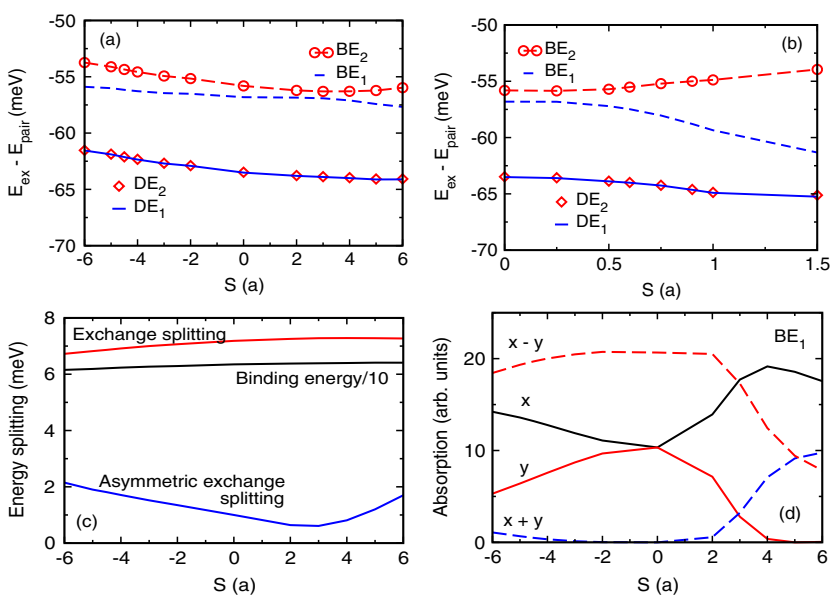

FIG. 4 (color online). Exciton fine structure for (a) biaxial deformation and (b) shearing bend. (c) Energy splittings for biaxial deformation. (d) Absorption strength of the lowest BE for biaxial deformation and polarization along QD diagonals $(x+y, x-y)$ and QD sides parallel $(x)$ and perpendicular $(y)$ to the bend.

binding energy increases (decreases) for a biaxial deformation with $S>0(S<0)$ because the electron and hole are pushed more into (out of) the QD [see Fig. 2]. The binding increases for a shearing bend, even though the electron and hole are separated laterally, because the vertical electron-hole separation is reduced by the shearing bend. The BEs are split from the DEs by exchange interaction. The BEs are split by the AES. Binding energy and DE-BE exchange splitting vary slowly as mechanical strain is applied. However, mechanical strain significantly alters AES, with reduced AES and an apparent anticrossing for a biaxial deformation with $S>0$ and increased AES for a shearing bend.

When mechanical strain is applied, DEs remain dark and BEs remain bright, because DE transitions, with change in total $z$ spin $\Delta J_{z} \neq \pm 1$, are not strongly mixed with $\mathrm{BE}$ transitions with $\Delta J_{z}= \pm 1$. The two BEs remain orthogonally polarized after bending. However, BE polarization is rotated significantly by bending. In a flat bridge, BEs are polarized along QD diagonals, as in Fig. 4(d). For a biaxially deformed bridge, the lowest $\mathrm{BE}$ becomes polarized along the [100] bend for $S>0$ and approaches this polarization for $S<0$. For a shearing bend, the polarization of the lowest BE rotates instead toward [010], becoming perpendicular to the bend for $S \simeq 2 a$. Strain-induced distortion of the single-particle dipole moments cannot explain the rotation of exciton polarization. The polarizations of single particle transitions change only by about $10 \%$ for these strains (not shown here). As we will discuss, the strain reengineers the exciton exchange coupling to modify not only the exchange splitting but also the phase of the spin mixing that determines the polarization.

The BEs are mixtures of the two degenerate, $J_{z}= \pm 1$ pair states that are excited by circularly polarized light $\left(\Delta J_{z}= \pm 1\right)$. The (real) Coulomb matrix element $V_{\text {Coul }}$ determines the binding energy, but does not strongly mix $J_{z}$. DE-BE exchange splitting is determined by a (real) exchange matrix element which also conserves spin, $V_{\text {exch,sc }}$. AES between BEs is determined by the magnitude of the (complex) off-diagonal exchange interaction $V_{\text {exch,mix }}$ which does mix $J_{z} \cdot V_{\text {Coul }}$ and $V_{\text {exch,sc }}$ are much larger than $\left|V_{\text {exch,mix }}\right|$, but strain-induced changes in $V_{\text {Coul }}$ and $V_{\text {exch,sc }}$ are comparable to $\left|V_{\text {exch,mix }}\right|$, as shown in Fig. 5. $\Delta V_{\text {Coul }}, \Delta V_{\text {exch,sc }}$, and $\left|V_{\text {exch,mix }}\right|$ follow, respectively, the change in binding energy, the DE-BE exchange splitting, and the AES shown in Fig. 4(c). $V_{\text {Coul }}$ and $V_{\text {exch,sc }}$ vary smoothly under strain while $\left|V_{\text {exch,mix }}\right|$ shows the apparent anticrossing. This is a matrix element effect rather than a true anticrossing, because the exciton character does not switch near the anticrossing.

Because $J_{z}= \pm 1$ pair states are mixed to make BEs, the phase of mixing is determined by the phase of $V_{\text {exch,mix }}$ in Fig. 5. BE polarization is determined by the phase of the mixing. When phases for these pair states are chosen so that their optical dipole moments are proportional to $\hat{x} \pm$ $i \hat{y}$, then $V_{\text {exch,mix }}$ is imaginary for $S=0$, ensuring that BEs 

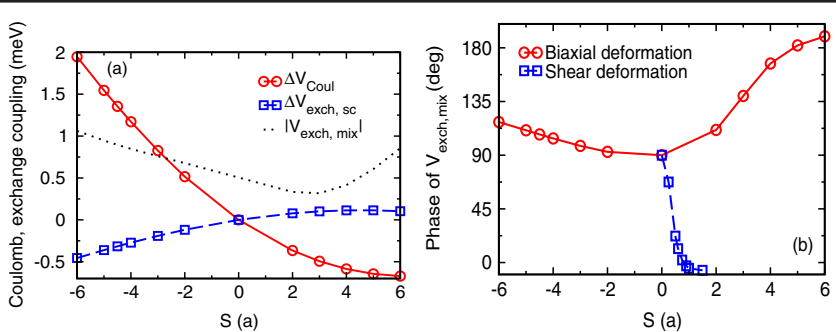

FIG. 5 (color online). (a) Change of $V_{\text {Coul }}, V_{\text {exch,sc }}$, and $\left|V_{\text {exch,mix }}\right|$ for a biaxial deformation. (b) Phase of $V_{\text {exch,mix }}$.

are polarized along QD diagonals in a flat bridge. When strain is applied, the phase of $V_{\text {exch,mix }}$ and the polarization rotate. The sign of $V_{\text {exch,mix }}$ determines whether polarization rotates toward $x$ or $y$.

A picture of why strain controls the phase of $V_{\text {exch,mix }}$ emerges by looking at Coulomb and exchange matrix elements. $V_{\text {Coul }}$ couples electron density at $\mathbf{r}\left[\rho_{e}(\mathbf{r})=\right.$ $\left|\phi_{e}(\mathbf{r})\right|^{2}$, for electron state $\left.\phi_{e}(\mathbf{r})\right]$ with hole density at $\mathbf{r}^{\prime}$, $\left[\rho_{h}\left(\mathbf{r}^{\prime}\right)=\left|\phi_{h}\left(\mathbf{r}^{\prime}\right)\right|^{2}\right] . V_{\text {Coul }}$ is real and spin independent. $V_{\text {exch,sc }}$ couples polarization density $P(\mathbf{r})$ at $\mathbf{r}$, with $P(\mathbf{r})=$ $\phi_{e}(\mathbf{r}) \phi_{h}(\mathbf{r})$, and the conjugate polarization density at $\mathbf{r}^{\prime}$, $P^{*}\left(\mathbf{r}^{\prime}\right)$. Thus, $V_{\text {exch,sc }}$ is real, depends mostly on the interaction weighted average of $|P(\mathbf{r})|\left|P\left(\mathbf{r}^{\prime}\right)\right|$ and weakly on the phase difference between $P(\mathbf{r})$ and $P\left(\mathbf{r}^{\prime}\right)$. In contrast, for $V_{\text {exch,mix }}$, the Kramers degeneracy of pairs with opposite spin ensures that the coupling is between $P(\mathbf{r})$ and $P\left(\mathbf{r}^{\prime}\right)$. $V_{\text {exch,mix }}$ and $V_{\text {exch,sc }}$ depend similarly on $|P(\mathbf{r})|$, but $V_{\text {exch,mix }}$ depends on the sum of the phases of $P(\mathbf{r})$ and $P\left(\mathbf{r}^{\prime}\right)$. Mechanical strain manipulates exciton binding energy and DE-BE exchange splitting by reshaping the electron and hole densities. The smooth variation of binding energy and exchange splitting reflect limited straininduced reshaping. The exciton polarization and phase of spin mixing are manipulated by changing the relative phase and orientation of the electron and hole pair. BE polarization varies for alloy QDs with the same composition but different atom distributions [41]. This also points to the connection between polarization, phase of spin mixing, and relative orientation of the electron and hole.

In conclusion, nanomechanical strain can be used to reengineer quantum dots. Electron and hole energies and distributions shift together or in opposite directions depending on how strain is applied, giving control to tune optical response. Strain can be used to transfer carriers between dots, giving a tool to interact qubits for quantum information processing. Changes in band gap, finestructure splitting, charge shifts, and polarization correlate to applied strain, giving signatures to gauge local strain. Internal strain from lattice mismatch, the nanomechanical strain, and internal readjustment to undo the applied strain must all be included to model strain effects. Most importantly, the applied strain can be used to manipulate mechanoexciton phase and fine structure, and rotate polar- ization, providing both energy and phase control to modify the inner workings of excitons.

*garnett.bryant@nist.gov

[1] O. Benson et al., Phys. Rev. Lett. 84, 2513 (2000).

[2] R. J. Young et al., Phys. Rev. B 72, 113305 (2005).

[3] R. M. Stevenson et al., Phys. Rev. B 73, 033306 (2006).

[4] K. Kowalik et al., Appl. Phys. Lett. 86, 041907 (2005).

[5] B. G. Gerardot et al., Appl. Phys. Lett. 90, 041101 (2007).

[6] M. E. Reimer et al., Phys. Rev. B 78, 195301 (2008).

[7] A. Muller et al., Phys. Rev. Lett. 101, 027401 (2008).

[8] T. Nakaoka et al., J. Appl. Phys. 94, 6812 (2003).

[9] J. R. Gell et al., Appl. Phys. Lett. 93, 081115 (2008).

[10] S. Seidl et al., Appl. Phys. Lett. 88, 203113 (2006).

[11] F. Ding et al., Phys. Rev. Lett. 104, 067405 (2010).

[12] M. Eichenfield et al., Nature (London) 459, 550 (2009).

[13] T. Zander et al., Opt. Express 17, 22452 (2009).

[14] T. Kipp et al., Phys. Rev. Lett. 96, 077403 (2006).

[15] S. Mendach et al., Appl. Phys. Lett. 88, 111120 (2006).

[16] X. Li, J. Phys. D 41, 193001 (2008).

[17] S. Vicknesh, F. Li, and Z. Mi, Appl. Phys. Lett. 94, 081101 (2009).

[18] K. L. Ekinci, Y. T. Yang, and M. L. Roukes, J. Appl. Phys. 95, 2682 (2004).

[19] K. Jensen, K. Kim, and A. Zettl, Nature Nanotech. 3, 533 (2008).

[20] R. H. Blick et al., New J. Phys. 9, 241 (2007).

[21] I. Mahboob and H. Yamaguchi, Nature Nanotech. 3, 275 (2008).

[22] M. S. Majdoub, P. Sharma, and T. Çağin, Phys. Rev. B 78, 121407 (2008).

[23] M. D. LaHaye et al., Science 304, 74 (2004).

[24] R. G. Knobel and A. N. Cleland, Nature (London) 424, 291 (2003).

[25] I. Wilson-Rae, P. Zoller, and A. Imamoǵlu, Phys. Rev. Lett. 92, 075507 (2004).

[26] K. Hammerer et al., Phys. Rev. Lett. 102, 020501 (2009).

[27] C. Rocke et al., Phys. Rev. Lett. 78, 4099 (1997).

[28] F. Alsina et al., Solid State Commun. 129, 453 (2004).

[29] M. M. de Lima, Jr. and P. V. Santos, Rep. Prog. Phys. 68, 1639 (2005).

[30] M. Kataoka et al., Phys. Rev. Lett. 98, 046801 (2007).

[31] T. Sogawa et al., Phys. Rev. B 80, 075304 (2009).

[32] O. D. D. Couto, Jr. et al., Nat. Photon. 3, 645 (2009).

[33] J. G. Diaz et al., Phys. Rev. B 75, 245433 (2007).

[34] W. Jaskólski et al., Phys. Rev. B 74, 195339 (2006).

[35] J. G. Diaz et al., Phys. Rev. B 74, 205309 (2006).

[36] K. Leung and K. B. Whaley, Phys. Rev. B 56, 7455 (1997).

[37] A. Franceschetti et al., Phys. Rev. B 60, 1819 (1999).

[38] S. Lee et al., Phys. Rev. B 63, 195318 (2001).

[39] G. Bester, S. Nair, and A. Zunger, Phys. Rev. B 67, 161306 (2003).

[40] R. Singh and G. Bester, Phys. Rev. Lett. 103, 063601 (2009).

[41] V. Mlinar and A. Zunger, Phys. Rev. B 79, 115416 (2009).

[42] T. Takagahara, Phys. Rev. B 62, 16840 (2000).

[43] C. Pryor et al., J. Appl. Phys. 83, 2548 (1998). 